\title{
Subject Index Vol. 35, 1997
}

Active avoidance 30 Acute-phase proteins 123 Adrenaline 205

Adrenocorticotropic hormone 73 Aging 11

Alpha-1 -acid glycoprotein 123 Alpha-1-antichymotrypsin 123 Alzheimer 95 Amantadine 16 Amplification factors 108 Anabolic/androgenic steroids 143 Antidepressant 91

drug 24 Antipsychotics 57 Anxiety 115 Arrhythmia 175 Attention 147

deficit hyperactivity disorder 178,181 Auditory oddball 191

vertex potential 51 Autism 1

Autopsy 95

Basal ganglia 36

Behaviour 200

Brain electric field maps 46

$$
\text { functional state } 46
$$

Cardiovascular morbidity 175

Carnitine 16

Cellular immunity 200

Cerebrospinal fluid 187

Childhood 51

Cholecystokinin 81

Chronic fatigue syndrome 16, 115

Circadian rhythms 84

Classification, rat 221

Clozapine 197

Cognition 51

Complement 181

Compliance 70

Conditioned fear 30

Connors Rating Scale 178

Cortisol 73, 205

Costs 67

CRF 200

Cyclandelate 132

Cyclic GMP 128

Dementia 132,187

Depression 11, 24, 95, 108, 115, 128

Diagnosis, schizophrenia 61

Diet 5

Dopamine 36

Dopaminergic activity 197

Drug infusion 211

Electroencephalogram(s) 91,95, 132,211 Electroencephalography 46 
Emotionality 205 ß-Endorphin 1 Epidemiology 57 ER in cognitive neuroscience 191 Event-related potentials 108 Fatigue 16 Fenfluramine 73 Fibromyalgia 115 Frequency bands 108 Frontal cortex 11

GABA 11

GABAergic steroids 143

Genetics 57

Global Dimensional Complexity 46

- $\quad$ Omega Complexity 46

Glucocorticoids 143

Growth hormone 79

Gum chewing 46

Healthcare 67 Heart rate 175 Holier ECG 175 5-HT receptors 79 Human brain 11

lymphocyte antigens 181

subjects 36 Hypnogram 84

Inferior colliculus 30 Interferon 128 Iron 178

Latent inhibition 30

Learning/attention disorders 51

Lithium potentiation of antidepressants 123

Major histocompatibility complex 181 Management 70 Melatonin 205

al-Microglobulin-like immunoreactive substance 187

Negative symptoms 64 Neural networks 221 Neuroactive steroids 147 Neuroleptic malignant syndrome 197 Neurosteroids 147 Neurotransmitters 200

Nicotine 102 Nipecotic acid 11

Nonconvulsive status epilepticus 91 Noradrenaline 205

Occipital EEG 102 Oddball 51 
P300 108

Panic disorder 175

Parkinson's disease 73,79,187

Pedunculopontine tegmental nucleus 102

Peripheral blood mononuclear cells 1

Personality 205

Pervasive developmental disorders 1

Pharmaco-EEG 152

Pharmacoeconomics 67

Physostigmine 102

Polysomnogram 152

Progesterone 147

metabolism 143

Psychiatric diagnosis 95

Psychometry 132

Rating scales 64, 132 Relapse 70

REM sleep discrimination 221 ß-Rhythm 211

Schizophrenia 5, 57, 61, 64, 67, 70, 108, 191

Selective attention 191

Serotonergic system 73

Serotonin 5

Sigma ligand JO 1784200

Single dose 152

Sleep 115

apnea 115

spindles 211 Sleep-wake stages 221 Slow wave sleep 152 Smell 46

Soluble interleukin-2 receptor 128

State space trajectory 46

Stress 200,205

Study, double-blind, placebo-controlled 24

Subtypes, schizophrenia 61

Suicide 11

Sumatriptan 79

Taste 46 Testosterone 205 Theanine 46 Thermogram 84 Theta rhythm 102 Tianeptine 24 Time perception 36 Treatment 132 Tryptophan 5 Two-term function 84

Ulinastatin-like immunoreactive substance 187 Ultradian rhythms 84 Unrestrained behaviour, rat 102 Urinary excretion 205

Zopiclone 152

K A $1 \backslash G$ E $1 \backslash$ (C) 1997 S. Karger AG, Basel

E-Mail karger@karger.ch Fax+ 41613061234 http://www. karger. ch 